\title{
The Comparison of Magnetic Resonance Imaging/ Transrectal Ultrasound Fusion Biopsies with Conventional Transrectal Biopsies in Prostate Cancer Detection
}

\author{
(1) Dursun Baba, (1) Ahmet Yıldırım Balık, (1) Alpaslan Yüksel, (1) Yusuf Şenoğlu \\ Düzce University Faculty of Medicine, Department of Urology, Düzce, Turkey
}

\begin{abstract}
Objective: The conventional technique for histological prostate cancer diagnosis is transrectal ultrasound (TRUS)-guided random sampling of the peripheral prostate zone. However, due to method insufficiency and recent developments in prostate imaging, new biopsy methods were introduced. This study aimed to evaluate prostate cancer detection rates by the standard and magnetic resonance (MR) fusion biopsy methods. The main purpose of our study is to mutually evaluate prostate cancer detection rates and results of standard and cognitive MR fusion biopsy methods and share our experiences in this process.

Materials and Methods: Patients, who underwent prostate biopsy due to elevated serum prostate-specific antigen levels (>4ng/mL) and/or suspicious rectal examination, were retrospectively evaluated. A total of 160 patients were included in the study between January 2018 and January 2021 . Patients were divided into two groups according to the applied method, as standard biopsy (SB) and MR fusion biopsy.

Results: Prostate cancer was reported in $25(31.3 \%)$ of 80 patients who underwent SB, wherein $20(25 \%)$ were determined with clinically significant cancer. Prostate cancer was reported in 30 (37.5\%) of 80 patients who underwent MR fusion biopsy, wherein 25 (31\%) were reported as clinically significant cancer. A statistically significant difference was found in detecting prostate cancer and clinically significant prostate cancer when the prostate imaging-Reporting and Data System $(3,4,5)$ scores were compared with each other $(p<0.05, p=0.00)$. The additional SB to MR-targeted fusion biopsy was statistically significant in prostate cancer diagnosis $(p=0.01, p<0.05)$

Conclusion: The additional SB to targeted biopsy increased the detection rate of clinically significant prostate cancer. Larger randomized studies are needed to reach a consensus on the ideal biopsy technique.

Keywords: Prostate cancer, MRI-ultrasound fusion, prostate biopsy, targeted biopsy

\section{Introduction}

Prostate cancer is the second most common cancer in men (1). For histological diagnosis, 10-12 focal biopsy accompanied using transrectal ultrasound (TRUS) is still the standard (conventional) biopsy method (2). The shortcomings of this method include the increased number of biopsies, the missed diagnosis of clinically significant cancer, and the detection of clinically insignificant cancer. Prostate biopsy diagnosis with standard biopsy (SB) is $25 \%-40 \%$ and $20 \%-25 \%$ of clinical cancers are missed. In addition, a certain rate of clinically insignificant cancers are detected $(3,4,5)$. Clinically insignificant cancer detection causes unnecessary treatments, and, on contrary, missed clinically important cancer causes diagnosis delay. Therefore, a conventional biopsy is questioned in prostate cancer diagnosis and new methods were investigated to increase the diagnostic quality of biopsy. Especially the use of magnetic resonance (MR)

in prostate imaging has introduced targeted biopsy (TB) studies. The purpose of integrating multiparametric MRI (mpMRI) into biopsy is to eliminate the deficiency in detecting clinically important cancer, detect lesions in the anterior prostate, which are difficult to sample especially in the conventional biopsy and obtain a TB from the detected lesion. Three different targeted prostate biopsy methods are defined: MR-US fusion biopsy, realtime MRI fusion-guided biopsy, and direct-MRI-guided biopsy $(3,6)$. The rates of prostate cancer detection vary between $38 \%$ $80 \%$ using the TB methods (7). The MR-USG fusion biopsy protocol lesions were detected by images obtained with mpMRI matched with TRUS image and TB sampling is performed under the guidance of TRUS. As one of the TB techniques, the MRUSG fusion biopsy is advantageous because it is cheap and fast, whereas the lack of standardization and experience is a disadvantage $(3,4)$.
\end{abstract}


This study aimed to mutually evaluate prostate cancer detection rates and results of standard and cognitive MFB methods and share our experiences in this process. The comparison of these two methods in the prostate biopsy procedure will guide and be beneficial for all clinicians, especially for urologists who perform prostate biopsy and radiologists who interpret mpMRI.

\section{Materials and Methods}

Approval for the study was obtained from Düzce University Ethics Committee (approval no: 2021/51, date: 01.03.2021). Between January 2018 and January 2021, patients who underwent prostate biopsy because of elevated serum prostatespecific antigen (PSA) levels ( $>4 \mathrm{ng} / \mathrm{mL}$ ) and/or suspicious rectal examination findings were retrospectively evaluated in our clinic. A total of 160 patients were included in the study. Patients were divided into two groups as patients who underwent SB $(n=80)$ and patients who underwent MFB $(n=80)$. Patients of the MFB group underwent TB from suspicious lesions obtained from mpMRI images and fusion SB (FSB) with standard 12 core. Patients who had previously undergone prostate biopsy for any reason and patients with PSA values above $20 \mathrm{ng} / \mathrm{mL}$ were excluded from the study. Prostate imaging was performed with mpMRI before the patient underwent fusion biopsy.

Pre-biopsy urine culture was performed in all patients. Those with positive urine culture were treated and urine culture sterility was achieved. Patients using anticoagulants were consulted to the relevant department and, without contraindications, short-acting anticoagulant treatment was initiated before the procedure. An appropriate dosage of antibiotic prophylaxis (Gentamicin, Genta® ampoule IM) was administered to patients $30 \mathrm{~min}$ before the procedure, and then sterile lubricating gel (Cathajell® $12.5 \mathrm{~g}$ ), which is also a rectal analgesia lubricant, was rectally applied. In a fetal position (lateral decubitus position), $10 \mathrm{cc}$ (5 cc each side) of local anesthesia (Citanest ${ }^{\circledR}$ $2 \%$ ) was applied between the prostate and seminal vesicles with a $20 \mathrm{G} 25 \mathrm{~cm}$ aspiration needle under the guidance of a TRUS probe. Twelve focal biopsies were systematically taken from 80 patients who underwent conventional biopsy. The biopsy samples were fixed with formol and sent in Eppendorf tubes for histopathological examination. After procedure completion, patients were followed up in the service for 3 hours and were discharged by prescribing ciprofloxacin $500 \mathrm{mg}$ oral tablet twice a day after a spontaneous micturition.
The same preparations were made in patients in the MFB group. For MpMRI, 3 Tesla Siemens AG MagnetomR Skyra (Germany) magnet MRI device was used. T2-weighted imaging was performed in the coronal, axial, and sagittal planes. In addition, diffusion-weighted imaging and dynamic contrast MRI sequences were used. US and MR images were matched regarding information such as zonal anatomy, prostate cyst, and prominent nodule using navigation ( $\left.\mathrm{V}-\mathrm{Nav}{ }^{\circledR}\right)$ system compatible with the ultrasound device (Logiq s8 GE Healthcare ${ }^{\circledR}$ ). Biopsy was taken from the suspicious prostate imaging-reporting and data system (PI-RADS 3 or above) lesions interpreted by the radiology. Conventional biopsy was also performed for all of these patients after the lesion biopsy. Biopsy results reported as malignant were classified as clinically significant or insignificant cancer according to Epstein Criteria defined by Epstein et al. (5). Patients with a PSA density of $<0.15 \mathrm{ng} / \mathrm{mL}$, a Gleason score reported as $3+3$, clinical staging of $\mathrm{T} 1 \mathrm{c}$, a positive core count of $<3$, and a cancer rate of $<50 \%$ per core were defined as clinically insignificant prostate cancer.

\section{Statistical Analysis}

In the comparisons between groups, continuous variables were examined with the Independent $t$ or Mann-Whitney $U$ test depending on data distribution, and categorical variables were examined with appropriate cross-table statistics. The Wilcoxon paired two-sample tests were used for dependent group comparisons. A comparison of qualitative variables was made using the chi-square and McNemar tests. Results were evaluated at a 95\% confidence interval and significance level of $p<0.05$. The Pearson or Spearman correlation analysis was used for continuous correlation analysis between variables.

\section{Results}

The mean PSA of patients who underwent SB was $7.21 \mathrm{ng} / \mathrm{mL}$, and the mean PSA of the MFB patients was $6.54 \mathrm{ng} / \mathrm{mL}$, and the difference between these two groups was not statistically significant. Malignant digital rectal examination (DRE) revealed findings of $53.5 \%$ of patients with conventional biopsy and $46.5 \%$ of patients with MFB and the difference was not significant $(p>0.05)$. The demographic characteristics of the MFB and SB patients are detailed in Table 1.

In the SB group, 38 patients had an abnormal DRE, wherein 15 (39\%) had prostate cancer and $14(36 \%)$ of these 15 patients

\begin{tabular}{|l|l|l|l|l|}
\hline \multicolumn{2}{|l|}{ Table 1. SB and MFB demographic features } & \multicolumn{2}{l|}{ MFB } \\
\hline & \multicolumn{1}{|l|}{ SB } & \multicolumn{2}{l|}{$\begin{array}{l}\text { Standard } \\
\text { deviation }\end{array}$} \\
\cline { 2 - 5 } & Average-range & $\begin{array}{l}\text { Standard } \\
\text { deviation }\end{array}$ & Average-range & 6.64 \\
\hline Age (years) & $64.96(50-81)$ & 7.46 & $63.79(48-76)$ & 3.089 \\
\hline Serum PSA $(\mathrm{ng} / \mathrm{mL})$ & $7.21(0.95-19.3)$ & 4.225 & $6.54(1.1-17)$ & 0.843 \\
\hline Serum free PSA $(\mathrm{ng} / \mathrm{mL})$ & $1.49(0.166-0.49)$ & 1.097 & $1.34(0.24-4.90)$ & $37.54(18-240)$ \\
\hline Prostate volume $(\mathrm{mL})$ & $59.6(20-210)$ & 35.359 & $0.144(0.25-0.6)$ & 0.099 \\
\hline PSA density $\left(\mathrm{ng} / \mathrm{mL}^{2}\right)$ & $0.149(0.02-0.75)$ & 0.115 & $14.63(13-17)$ \\
\hline Total number of cores & $12(12)$ & 0 & & 0.986 \\
\hline SB: Standart biopsy, MFB: MRG-US fusion biopsy, PSA: Prostate-specific antigen & & \\
\hline
\end{tabular}


had clinically significant prostate cancer. Clinically significant prostate cancer was detected in 6 (14\%) of the patients with normal DRE. Thirty (37.5\%) of 80 patients in the FSB group were reported as prostate cancer, and 25 (31\%) of these patients were reported as clinically significant cancer. In the FSB group, 33 of 80 patients had malignant DRE findings, wherein 22 (66.6\%) had prostate cancer. A statistically significant correlation was found between the DRE and fusion biopsy pathology results. $(p<0.05, p=0.01$ correlation coefficient: 0.5$)$

Prostate cancer was detected in the TB of 6 (12.5\%) of 48 patients who had PI-RADS 3 lesions on mpMRI. Prostate cancer was detected in 11 (50\%) of 22 patients with PI-RADS 4 lesions. Prostate cancer was detected in 6 of 10 (60\%) patients with PIRADS 5 lesions. Clinically significant cancer was detected in 21 of 23 (95\%) of patients who had PI-RADS 3,4, and 5 lesions with TB (Table 2). A statistically significant difference was found when the PI-RADS 3, 4, and 5 scores were compared with each other in detecting prostate cancer and clinically significant prostate cancer $(p<0.05, p=0.00)$. Clinically significant prostate cancer was reported in $90 \%$ of patients with PI-RADS 4 lesions and $100 \%$ PI-RADS 5 lesions.

While 20 of 80 (25\%) patients in the SB group had clinically significant prostate cancer, 25 (31.8\%) of 80 patients in the MFB group have prostate cancer. When the MFB group is evaluated as FSB and TB separately; 21 (28.7\%) of 80 patients who underwent FSB biopsy have clinically significant prostate cancer, whereas 23 patients have clinically significant prostate cancer in the TB group. In the TB group, 21 (86.9\%) of 23 patients

\begin{tabular}{|l|l|l|l|l|}
\hline \multicolumn{5}{|l|}{ Table 2. Fusion biopsy PI-RADS classification of prostate cancer } \\
\hline PI-RADS score & 3 & 4 & 5 & Total \\
\hline Number of patients & $48(100 \%)$ & $22(100 \%)$ & $10(100 \%)$ & 80 \\
\hline Have prostate Ca & $6(12.5 \%)$ & $11(50 \%)$ & $6(60 \%)$ & 23 \\
\hline Prostate Ca no & $42(87.5 \%)$ & $11(50 \%)$ & $4(40 \%)$ & 57 \\
\hline $\begin{array}{l}\text { Clinically significant } \\
\text { cancer }\end{array}$ & $5(83.3 \%)$ & $10(90.9 \%)$ & $6(100 \%)$ & 21 \\
\hline $\begin{array}{l}\text { Clinically unsignificant } \\
\text { cancer }\end{array}$ & $1(16.7 \%)$ & $1(9.1 \%)$ & $0(0 \%)$ & 2 \\
\hline PI-RADS: Prostate imaging-reporting and data system \\
\hline
\end{tabular}

\begin{tabular}{|c|c|c|c|}
\hline & \multirow{2}{*}{$\begin{array}{l}\text { SB } \\
\text { n (\%) }\end{array}$} & \multicolumn{2}{|l|}{ MFB } \\
\hline & & $\begin{array}{l}\text { FSB } \\
\text { n (\%) }\end{array}$ & $\begin{array}{l}\text { TB } \\
\mathrm{n}(\%)\end{array}$ \\
\hline Benign & $\begin{array}{l}55 \\
(68.8 \%)\end{array}$ & $\begin{array}{l}50 \\
(62.5 \%)\end{array}$ & $\begin{array}{l}57 \\
(71.2 \%)\end{array}$ \\
\hline Prostat cancer & $\begin{array}{l}25 \\
(31.3 \%)\end{array}$ & $\begin{array}{l}30 \\
(37.6 \%)\end{array}$ & $\begin{array}{l}23 \\
(28.7 \%)\end{array}$ \\
\hline $\begin{array}{l}\text { Clinically significant prostate } \\
\text { cancer }\end{array}$ & $\begin{array}{l}20 \\
(25 \%)\end{array}$ & $\begin{array}{l}25 \\
(31.8 \%)\end{array}$ & $\begin{array}{l}21 \\
(26.2 \%)\end{array}$ \\
\hline $\begin{array}{l}\text { Clinically unsignificant } \\
\text { prostate cancer }\end{array}$ & $\begin{array}{l}5 \\
(6.3 \%)\end{array}$ & $\begin{array}{l}5 \\
(6.3 \%)\end{array}$ & $\begin{array}{l}2 \\
(2.5 \%)\end{array}$ \\
\hline Total & $\begin{array}{l}80 \\
(100 \%)\end{array}$ & $\begin{array}{l}80 \\
(100 \%)\end{array}$ & $\begin{array}{l}80 \\
(100 \%)\end{array}$ \\
\hline
\end{tabular}

SB: Standard biopsy, MFB: MRI-US fusion biopsy, FSB: Fusion standard biopsy, TB: Targeted biopsy had clinically significant cancer (Table 3). When patients in MFB were separately evaluated as FSB and TB group, additional SB to the TB obtained from the lesion provided an approximately $8.9 \%$ advantage in prostate cancer diagnosis. This superiority was statistically significant $(p=0.01, p<0.05)$. The method with the highest number of prostate cancer and clinically significant prostate cancer was MFB. However, no significant difference was found between SB and MFB in prostate cancer or clinically significant prostate cancer detection $(p=0.253, p>0.05)$.

The comparison of SB and FSB revealed 25 (31.2\%) of 80 patients who underwent SB and 28 (35\%) of 80 patients who underwent FSB were diagnosed with prostate cancer. This difference was not statistically significant. ( $p=0.737)$. In $2(2.5 \%)$ patients, cancer was not detected by SB but was detected by TB. However, only one was clinically important cancer. When DRE findings were compared in SB and MFB groups, the cancer detection rate in the MFB group was statistically significant in patients with abnormal DRE $(p<0.05$, correlation coefficient: 0.5$)$.

\section{Discussion}

Biopsy methods used for prostate cancer diagnosis changed with technological developments. Due to TRUS-guided biopsy deficiencies, which was the SB method for a long time, new techniques were investigated. More sensitive methods are investigated due to the low sensitivity of ultrasound in detecting prostate gland lesions, skipping the diagnosis of clinically important cancer, and detecting clinically insignificant cancer. A cancer diagnosis is missed at a rate of approximately $33 \%$ in standard prostate biopsies performed under US guidance $(8,9)$. The use of mpMRI increased in recent years due to the advantages of showing the prostate anatomy more clearly, detecting the intraprostatic lesion more easily, showing the spread of lesion to the extracapsular region more clearly, and detecting small lesions, and has become a guide for targeted procedures in prostate biopsy. Therefore, targeted biopsies increased their popularity in detecting clinically important cancer.

In studies, cancer detection rates vary between $25 \%-40 \%$ in SB and between $38 \%-80 \%$ in targeted biopsies $(3,4,5,7)$. Similar results in cancer detection rates in the literature were found in our study (SB: $31.2 \%$, MFB: $35 \%$ ). No significant difference was found between the two methods, especially in patients who underwent the first biopsy.

Especially in patients with abnormal rectal examination findings, the rate of cancer detection by fusion biopsy was higher than SB. Therefore, prostatic imaging and subsequent fusion biopsy were superior to SB in diagnosing patients with rectal examination findings.

MRI-US fusion biopsy was not superior in detecting prostate cancer compared to SB in the meta-analysis that examined 16 studies; however, it was reported to have a higher rate of clinically significant prostate cancer detection and a lower rate of clinically insignificant prostate cancer detection (4). Other similar studies revealed that high-risk cancer at a higher rate of up to $30 \%$ and a lower rate of low-risk cancer are detected with fusion biopsy compared to SB $(3,10)$. In our study, fusion biopsy was numerically superior to SB in both detecting prostate cancer 
and detecting clinically significant prostate cancer; however, this difference was not statistically significant, due to insufficient sample size. Therefore, the insufficient sample reveals the limitations of the study.

When the subgroups of patients who underwent fusion biopsy were examined, a statistically significant prostate cancer detection rate was found when the SB was added to the TB compared to those who had only TB. Therefore, our study supports the idea that an SB should always be added to TB in patients undergoing fusion biopsy. TB taken from the lesion detected in mpMRI is advantageous in studies; however, adding a standard systematic biopsy to patients with TB is controversial (11). However, the study conducted by Siddiqui et al. (10). Revealed that MRI fusion biopsy was more successful in detecting clinically significant cancer than SB, while it was reported that the addition of SB to fusion biopsy also increased the diagnosis of clinically insignificant prostate cancer. Therefore, comprehensive studies investigating the clinical significance of prostate cancers detected by SB in addition to fusion are necessary. Our study compared the MRI-US fusion biopsy and SB, which found no statistically significant difference in detecting prostate cancer and clinically significant prostate cancer.

As the PI-RADS score determined by MpMRI increased, the rate of prostate cancer and clinically significant prostate cancer detection increased in a statistically significant way. However, this increase was significant, especially in patients with PI-RADS scores of 4-5, whereas not statistically significant in patients with PI-RADS scores of 3 . Therefore, MR-US fusion biopsy should be recommended primarily for diagnostic success, especially in patients with PI-RADS 4-5 lesion scores. In this context, comprehensive studies are needed on whether MR fusion or SB will be performed in patients with PI-RADS 3 lesion scores. The EAU 2019 guidelines recommend mpMRI with a weak recommendation before biopsy in patients who have not previously undergone a biopsy, whereas the EAU 2020 guideline strongly recommends imaging at the level of $1 \mathrm{~A}$ evidence. The EAU 2020 guideline strongly recommends a standard 12-core biopsy in addition to the TB if PI-RADS 3 or more lesions are detected on MRI at the level of evidence $2 \mathrm{a}$. $(12,13)$. Therefore, the PI-RADS score, which is evaluated by radiologists before biopsy, is very important.

\section{Study Limitations}

The number of patients in the study was small, thus a large sample size was not achieved, which created numerical differences; however, no statistically significant difference was found in some comparisons. The number of cores taken from patients who underwent TB was much lower than the number of cores taken in standard and fusion biopsy, making the calculation and comparison of rates of cancer capture per core difficult.

\section{Conclusion}

Improvement in mpMRI imaging, increase in experience of lesion evaluation, and MRI-USG fusion biopsy technique will contribute to the diagnosis, treatment, and follow-up of prostate cancer. No statistically significant difference was found between fusion biopsy and SB in detecting prostate cancer in our study; however, numerically more prostate cancer and clinically significant cancers were detected in fusion biopsy. Therefore, TB alone is insufficient, thus an SB must be added. Larger and randomized studies are needed to rule out clinically insignificant cancers among prostate cancers and create a consensus on the biopsy technique application.

\section{Acknowledgements}

Publication: The results of the study were not published in full or in part in form of abstracts.

Contribution: There is not any contributors who may not be listed as authors.

Conflict of Interest: No conflict of interest was declared by the authors.

Financial Disclosure: The authors declared that this study received no financial support.

\section{Ethics}

Ethics Committee Approval: Approval for the study was obtained from Düzce University Ethics Committee (approval no: 2021/51, date: 01.03.2021).

Informed Consent: Retrospective study.

Peer-review: Externally peer-reviewed.

\section{Authorship Contributions}

Critical Review: A.Y., Y.Ş., Supervision: D.B., A.Y., Y.Ş., Concept: D.B., Design: D.B., Data Collection or Processing: A.Y.B., Analysis or Interpretation: A.Y.B., Literature Search: A.Y.B., Writing: D.B., A.Y.B.

\section{References}

1. Arnold M, Karim-Kos HE, Coebergh JW, et al. Recent trends in incidence of five common cancers in 26 European countries since 1988: analysis of the European Cancer Observatory. Eur J Cancer 2015;51:1164-1187.

2. Dasgupta P, Davis J, Hughes S. NICE guidelines on prostate cancer 2019. New Jerset: Wiley Online Library; 2019.

3. Puech $P$, Rouvière $O$, Renard-Penna $R$, et al. Prostate cancer diagnosis: multiparametric MR-targeted biopsy with cognitive and transrectal US-MR fusion guidance versus systematic biopsy--prospective multicenter study. Radiology 2013;268:461-469.

4. Schoots IG, Roobol MJ, Nieboer D, et al. Magnetic resonance imaging-targeted biopsy may enhance the diagnostic accuracy of significant prostate cancer detection compared to standard transrectal ultrasound-guided biopsy: a systematic review and metaanalysis. Eur Urol 2015;68:438-450.

5. Epstein II, Walsh PC, Carmichael M, et al. Pathologic and clinical findings to predict tumor extent of nonpalpable (stage T1c) prostate cancer. Jama 1994;271:368-374.

6. Presti JC. Prostate biopsy strategies. Nat Clin Pract Uro 2007;4:505-511.

7. Brown AM, Elbuluk $\mathrm{O}$, Mertan $\mathrm{F}$, et al. Recent advances in image-guided targeted prostate biopsy. Abdom Imaging 2015;40:1788-1799.

8. Bipat S, van Leeuwen MS, Comans EF, et al. Colorectal liver metastases: CT, MR imaging, and PET for diagnosis-meta-analysis. Radiology 2005;237:123-131.

9. Rabbani F, Stroumbakis N, Kava BR, et al. Incidence and clinical significance of false-negative sextant prostate biopsies. J Urol 1998; 159:1247-1250. 
10. Siddiqui MM, Rais-Bahrami S, Turkbey B, et al. Comparison of MR/ ultrasound fusion-guided biopsy with ultrasound-guided biopsy for the diagnosis of prostate cancer. JAMA 2015;313:390-397.

11. Norris JM, Kinnaird A, Margolis DJ, et al. Developments in MRItargeted prostate biopsy. Curr Opin Urol 2020;30:1-8.
12. Mottet N, Van den Bergh R, Briers E. EAU guidelines: prostate cancer 2019. Eur Urol 2019;76:868-873.

13. Mottet N, van den Bergh RC, Briers E, et al. EAU-EANM-ESTROESUR-SIOG guidelines on prostate cancer-2020 update. part 1: screening, diagnosis, and local treatment with curative intent. Eur Urol 2021;79:243-262. 